\title{
Islet autoantibody phenotypes and incidence in children at increased risk for type 1 diabetes
}

\author{
Eleni Z. Giannopoulou ${ }^{1}$. Christiane Winkler ${ }^{1,2} \cdot$ Ruth Chmiel $^{1} \cdot$ Claudia Matzke $^{1}$. \\ Marlon Scholz $^{1}$ - Andreas Beyerlein ${ }^{1}$ - Peter Achenbach ${ }^{1,2} \cdot$ Ezio Bonifacio $^{3,4,5}$. \\ Anette-G. Ziegler ${ }^{1,2}$
}

Received: 28 January 2015 / Accepted: 31 May 2015 /Published online: 3 July 2015

(C) Springer-Verlag Berlin Heidelberg 2015

\begin{abstract}
Aims/hypothesis Autoantibodies that precede type 1 diabetes frequently develop in early childhood and target distinct beta cell proteins. The aim of this study was to determine the heterogeneity of islet autoantibody development and fate. Methods The ages of development of insulin autoantibodies (IAA) and GAD autoantibodies (GADA), followed by multiple islet autoantibodies and progression to diabetes were examined in 2,441 children participating in two German birth cohorts.

Results In 218 children who developed islet autoantibodies, the first islet autoantibody-positive sample was characterised
\end{abstract}

Ezio Bonifacio and Anette-G. Ziegler are joint senior authors on this paper.

Electronic supplementary material The online version of this article (doi:10.1007/s00125-015-3672-y) contains peer-reviewed but unedited supplementary material, which is available to authorised users.

Anette-G. Ziegler

anette-g.ziegler@helmholtz-muenchen.de

1 Institute of Diabetes Research, Helmholtz Zentrum München and Forschergruppe Diabetes, Klinikum rechts der Isar, Technische Universität München, Ingolstaedter Landstr. 1, 85764 Neuherberg, Germany

2 Forschergruppe Diabetes e.V., Neuherberg, Germany

3 Center for Regenerative Therapies Dresden, Faculty of Medicine, Technische Universität, Dresden, Germany

4 Paul Langerhans Institute of the Helmholtz Zentrum München at University Clinic Carl Gustav Carus, Technische Universität Dresden, Dresden, Germany

5 Institute of Diabetes and Obesity, Helmholtz Zentrum München, Neuherberg, Germany by single IAA in 80 (37\%), multiple islet autoantibodies in 68 (31\%) and single GADA in 63 (29\%) children. Of the children who were single antibody positive at seroconversion, 35 (44\%) IAA-positive and 15 (24\%) GADA-positive children developed multiple islet autoantibodies. Single persistent antibodies had heterogeneous affinities; GADA were also heterogeneous in their binding to N-terminally truncated GAD65 and in an ELISA. Progression to diabetes occurred in $>50 \%$ of children within 10 years in all groups that developed multiple islet autoantibodies and in $44 \%$ of children with persistent single high-affinity IAA or persistent single GADA that were positive in both a radiobinding assay and ELISA. The earliest autoantibody development was seen in children with single IAA that progressed to multiple islet autoantibodies or in those with persistent high-affinity single IAA, with a sharp peak in incidence observed at age 9 months. The peak incidence occurred at age 2 years for children who underwent seroconversion directly to multiple islet autoantibodies and at 5 years for children who first seroconverted to GADA and subsequently developed other autoantibodies. Seroconversion to low-affinity IAA or persistent single GADA occurred at a low incidence after the age of 9 months.

Conclusions/interpretation Children of different ages have differing susceptibilities to autoimmunisation against specific beta cell autoantigens.

Keywords Incidence $\cdot$ Islet autoantibodies $\cdot$ Type 1 diabetes

$\begin{array}{ll}\text { Abbreviations } \\ \text { DIPP } & \text { Type 1 Diabetes Prediction and Prevention } \\ \text { GADA } & \text { GAD autoantibodies } \\ \text { IA-2 } & \text { Insulinoma-associated antigen } 2 \\ \text { IA-2A } & \text { Insulinoma-associated antigen } 2 \text { autoantibodies } \\ \text { IAA } & \text { Insulin autoantibodies }\end{array}$


RBA Radiobinding assay

TEDDY The Environmental Determinants of Diabetes in the Young

ZnT8 Zinc transporter 8

ZnT8A Zinc transporter 8 autoantibodies

\section{Introduction}

The clinical onset of type 1 diabetes is usually preceded and characterised by circulating autoantibodies against the beta cell antigens insulin (IAA), GAD (GADA), insulinassociated antigen-2 (IA-2; IA-2A), and/or zinc transporter-8 (ZnT8; ZnT8A) [1, 2]. These autoantibodies may develop throughout childhood, but seroconversion is most frequent from 6 months to 2 years of age [3].

Seroconversion typically involves IAA and/or GADA, and is often followed quickly by expansion to multiple islet autoantibodies [4], an event that defines an asymptomatic stage of type 1 diabetes. Over $80 \%$ of children with multiple islet autoantibodies progress to symptomatic, insulin-requiring diabetes within 15 years [2]. In contrast, only a minority of the children who remain positive for a single islet autoantibody develops type 1 diabetes [2]. There is heterogeneity in the IAA and GADA found in children who remain single autoantibody-positive. Some of the IAA and GADA are typical of the high-affinity autoantibodies seen in children who have multiple autoantibodies, and others are low affinity or react only against atypical epitopes [5, 6].

We have been able to track the appearance and fate of islet autoantibodies in children for over 20 years [2]. In this study, we defined different phenotypes of IAA or GADA based on their order of appearance, their expansion to multiple islet autoantibodies, and their affinity or epitope specificity. We related islet autoantibody phenotype to the age of autoantibody appearance and risk of clinical diabetes. The findings suggest differences in the timing of insulin and GAD autoimmunity, and have implications for both the pathogenesis and prediction of type 1 diabetes.

\section{Methods}

Study population Data from two ongoing German birth cohorts of individuals with a family history of type 1 diabetes born between 1989 and 2000 (BABYDIAB [7]) and between 2000 and 2006 (BABYDIET [8]) were combined for this analysis. Both studies aimed to prospectively examine the natural history of islet autoimmunity and type 1 diabetes. The BABYDIAB study recruited 1,650 children born to a mother or father with type 1 diabetes between 1989 and 2000 [7]. Between 2000 and 2006, the BABYDIET study recruited 791 children with a first-degree relative with type 1 diabetes. A subgroup of 150 children carrying high-risk HLA genotypes participated in the BABYDIET gluten intervention study (ClinicalTrials.gov NCT01115621) to investigate whether delay of exposure to gluten could reduce the risk of developing autoantibodies. The intervention failed to show an effect on islet autoantibody development and all participants continued with follow-up examinations according to the natural history protocol [8]. Detailed descriptions of the design of each study have been reported [7, 8]. The studies were approved by the ethics committee of Bavaria, Germany (Bayerische Landesärztekammer no. 95357 and LudwigMaximilians University no. 329/00, respectively) and performed in accordance with the principles of the Declaration of Helsinki, including the provision of written informed consent for all participants.

Islet autoantibodies (IAA, GADA, IA-2A and ZnT8A) were measured in venous blood samples obtained at scheduled visits: at birth, 9 months and 2, 5, 8, 11, 14, 17 and 20 years, and at 3-monthly visits from birth until the age of 3 years and yearly until the age of 14 years for the 150 children who participated in the gluten intervention study. Measurement of islet autoantibodies in these studies was performed using radiobinding assays (RBAs), as described elsewhere [9-11]. Islet autoantibody positivity was defined as the development of persistent autoantibodies against one or more of the antigens insulin, GAD65, IA-2 or ZnT8, with sample values above the 99th percentile of published population control children classified as positive. Persistence was defined as autoantibody positivity in at least two consecutive samples. Age at the first autoantibody-positive sample was considered the seroconversion age in children. Children were classified as multiple islet autoantibody-positive if they tested positive for more than one of the islet autoantibodies on at least one occasion. Islet autoantibody assays were evaluated according to the Diabetes Autoantibody Standardization Program [9-11] and type 1 diabetes was diagnosed according to ADA Expert Committee criteria [12].

In children who were positive for GADA in the absence of other islet autoantibodies, GADA were also assessed for GAD-binding affinity [5], ELISA (Medizym anti-GAD ELISA Kit, Medipan, Berlin, Germany) positivity, and their binding to in vitro transcribed/translated, ${ }^{35} \mathrm{~S}$-labelled human N-terminally truncated GAD65 (GADA $_{96-585}$, amino acids 96-585; construct provided by V. Lampasona, San Raffaele Institute, Milan, Italy; threshold for positivity, $\geq 22$ units $/ \mathrm{ml}$ ). Single GADA were categorised as RBA plus ELISA-positive or RBA-positive/ELISA-negative. In children who were positive for IAA in the absence of other islet autoantibodies, IAA were assessed for insulin-binding affinity, as previously described [6]. IAA affinity was expressed as reciprocal $K_{\mathrm{d}}$ values $(1 / \mathrm{mol})$ and categorised as high affinity $\left(\geq 10^{9} \mathrm{l} / \mathrm{mol}\right)$ or low affinity $\left(<10^{9} \mathrm{l} / \mathrm{mol}\right)$. 
$H L A-D R B 1, H L A-D Q A 1$ and $H L A-D Q B 1$ genotyping in the BABYDIAB and BABYDIET studies has been described elsewhere [13].

Statistical analysis Time-to-event analyses (Kaplan-Meier estimates) were used to examine progression from islet autoantibody seroconversion to type 1 diabetes. The period from the age of seroconversion to the age at diagnosis of diabetes or the age at last follow-up was used as the event time. The logrank test was used to compare progression to type 1 diabetes in children stratified by antibody phenotype.

In accordance with the visit schedule, antibody incidence (per 1,000 person-years) was calculated from samples obtained within the age intervals $0-18$ months (mean age of sample group, 0.85 years), $18-42$ months (mean age of sample group, 2.0 years), 42-78 months (mean age of sample group, 5.0 years), $78-114$ months (mean age of sample group, 8.0 years), $114-150$ months (mean age of sample group, 11.0 years), $150-186$ months (mean age of sample group, 14.0 years) and 186-204 months (mean age of sample group, 17.0 years), by dividing the number of children who had newly seroconverted to islet autoantibody positivity by the number of children followed at each interval [3]. SE calculations were based on the Cooper-Pearson methods. Incidences of different islet autoantibody types were compared using Fisher's exact test. All statistical analyses were performed using the IBM SPSS Statistics software (version 22.0, Chicago, IL, USA).

\section{Results}

The median follow-up for all 2,441 children was 9.5 years (interquartile range, 6.5-15.5 years) for a total of 30,214 person-years. During follow-up, 218 children developed persistent islet autoantibodies (electronic supplementary material [ESM] Fig. 1). These included 150 children who had one autoantibody and 68 children who had multiple islet autoantibodies in the first positive sample. Of the 150 children with one islet autoantibody at seroconversion, 56 developed multiple islet autoantibodies during follow-up.

Based on the islet autoantibodies present in the first positive sample and at follow-up, children were categorised into the following phenotypes: IAA at seroconversion progressing to multiple islet autoantibodies later ( $\mathrm{P} 1 ; n=35,16.1 \%)$; GADA at seroconversion progressing to multiple islet autoantibodies later (P2; $n=15,6.9 \%)$; multiple islet autoantibodies in the first positive sample (P3; $n=68,31.2 \%)$; single IAA ( $n=$ $45,20.6 \%)$ with a high-affinity antibody phenotype $(n=9$; P4), and with a low-affinity antibody phenotype ( $n=36$; P5); and single GADA $(n=48,22.0 \%)$ that was positive by ELISA ( $n=9 ; \mathrm{P} 6)$ or negative by ELISA $(n=38 ; \mathrm{P} 7)$. Of note, single GADA were heterogeneous in their binding to GAD in the different assays and in their affinities (ESM Table 1). Single GADA that were positive in both the RBA and ELISA were of high affinity $\left(>10^{9} 1 / \mathrm{mol}\right)$ and eight out of nine were also positive for N-terminally truncated GAD65 $96-585$. Most single GADA that were negative by ELISA were of low affinity (23/38), were negative against N-terminally truncated GAD65 $96-585$ (30/39) and included a large number that had binding properties consistent with two or more binding sites.

Islet autoantibody phenotype and type 1 diabetes risk A total of 82 islet autoantibody-positive children developed diabetes during follow-up. Progression to type 1 diabetes was observed in each of the groups with IAA or GADA phenotypes that included multiple islet autoantibodies (P1-P3), and in those who had single islet autoantibodies that were high affinity or, for GADA, ELISA-positive (P4, P6) (Fig. 1). The 10-year risk of type 1 diabetes was $56 \%$ (95\% CI $38 \%$, $74 \%$ ) in children who had IAA and developed multiple islet

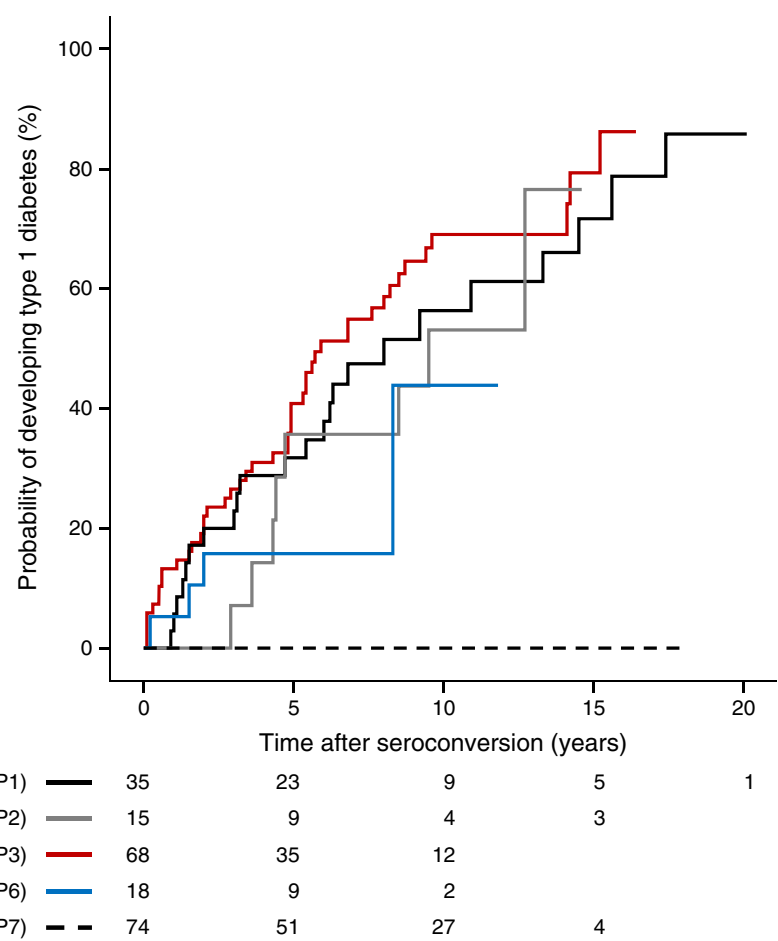

Fig. 1 Progression to type 1 diabetes from islet autoantibody seroconversion. Kaplan-Meier analysis of children who had IAA at seroconversion and subsequently developed other islet autoantibodies (P1; black line), GADA at seroconversion and subsequently developed other islet autoantibodies (P2; grey line), multiple islet autoantibodies in the first positive sample (P3; red line), single IAA with a high-affinity antibody phenotype or single GADA positivity in RBA and ELISA (P4, P6; blue line) and single IAA with a low-affinity antibody phenotype or single GADA positivity in RBA only (P5, P7; black dashed line). Progression to type 1 diabetes was different in children with a single low-risk islet autoantibody phenotype compared with a single high-risk islet autoantibody phenotype $(p<0.001)$ or multiple islet autoantibody phenotype $(p<0.001)$. Numbers under the $x$-axis indicate the number of children still being followed at each time point 
autoantibodies later (P1), 53\% (95\% CI 26\%, 80\%) in children who had GADA and developed multiple islet autoantibodies later (P2), 69\% (95\% CI 57\%, 81\%) in children who had multiple islet autoantibodies in the first positive sample (P3) and 44\% (95\% CI 0.1\%, 91\%) in children who had single high-affinity IAA (P4) or single GADA that were RBA and ELISA-positive (P6). Type 1 diabetes risk did not differ between these four groups. In contrast, none of the 74 children with single low-affinity IAA or single GADA RBA-positive/ ELISA-negative antibodies (P5 or P7) developed diabetes during follow-up ( $p<0.001$ vs single high-risk islet autoantibody phenotype; $p<0.001$ vs multiple islet autoantibody phenotypes).

Incidence of islet autoantibody phenotype Seroconversion age was heterogeneous amongst groups with different phenotypes. The earliest peak incidence was observed for children who first developed IAA and later became multiple islet autoantibody-positive (P1: peak incidence at age 9 months, 8.7 [SE \pm 2.0 ] per 1,000 person-years; Fig. 2a), and children who developed single high-affinity IAA (P4: peak incidence at age 9 months, $1.9[ \pm 1.0]$ per 1,000 person-years; Fig. 2b). The peak incidence of seroconversion to multiple islet autoantibodies occurred at age 2 years (P3: $10.3[ \pm 2.0]$ per 1,000 person-years; Fig. 2a); in children who first seroconverted to GADA and subsequently developed other autoantibodies, the peak incidence occurred at age 5 years (P2: $1.7[ \pm 0.5]$ per 1,000 person-years; Fig. 2a). For children with single lowaffinity IAA (Fig. 2b) and single GADA (Fig. 2c), there was no clear peak incidence age: seroconversion occurred at a low incidence after age 9 months.

Overall, the incidence of GADA preceding other islet autoantibodies was lower during the first 5 years of life compared with the incidence of IAA preceding other islet autoantibodies $(p<0.001)$ or with multiple islet antibodies at seroconversion $(p=0.04)$. In addition, the incidence of single high- and low-affinity IAA phenotypes or a single GADA phenotype was lower during the first 5 years compared with multiple islet autoantibody phenotypes $(p<0.001)$.

\section{Incidence of high-risk islet autoantibody phenotypes} by HLA genotype Differences in the incidence and incidence peaks were observed relative to the $H L A D R-D Q$ genotype (Fig. 3). Amongst children with the HLA DR3/DR4-DQ8 (Fig. 3a) or DR4-DQ8/DR4-DQ8 (Fig. 3b) genotypes, seroconversion to IAA preceding other islet autoantibodies or single high-affinity IAA was the dominant autoantibody phenotype, and the peak incidence of seroconversion occurred at age 9 months for both genotypes. In contrast, amongst children with the HLA DR3/DR3 genotypes (Fig. 3c), seroconversion to GADA preceding other islet autoantibodies or single RBAand ELISA-positive GADA was the dominant phenotype, and the peak incidence of seroconversion occurred at age 2 years.
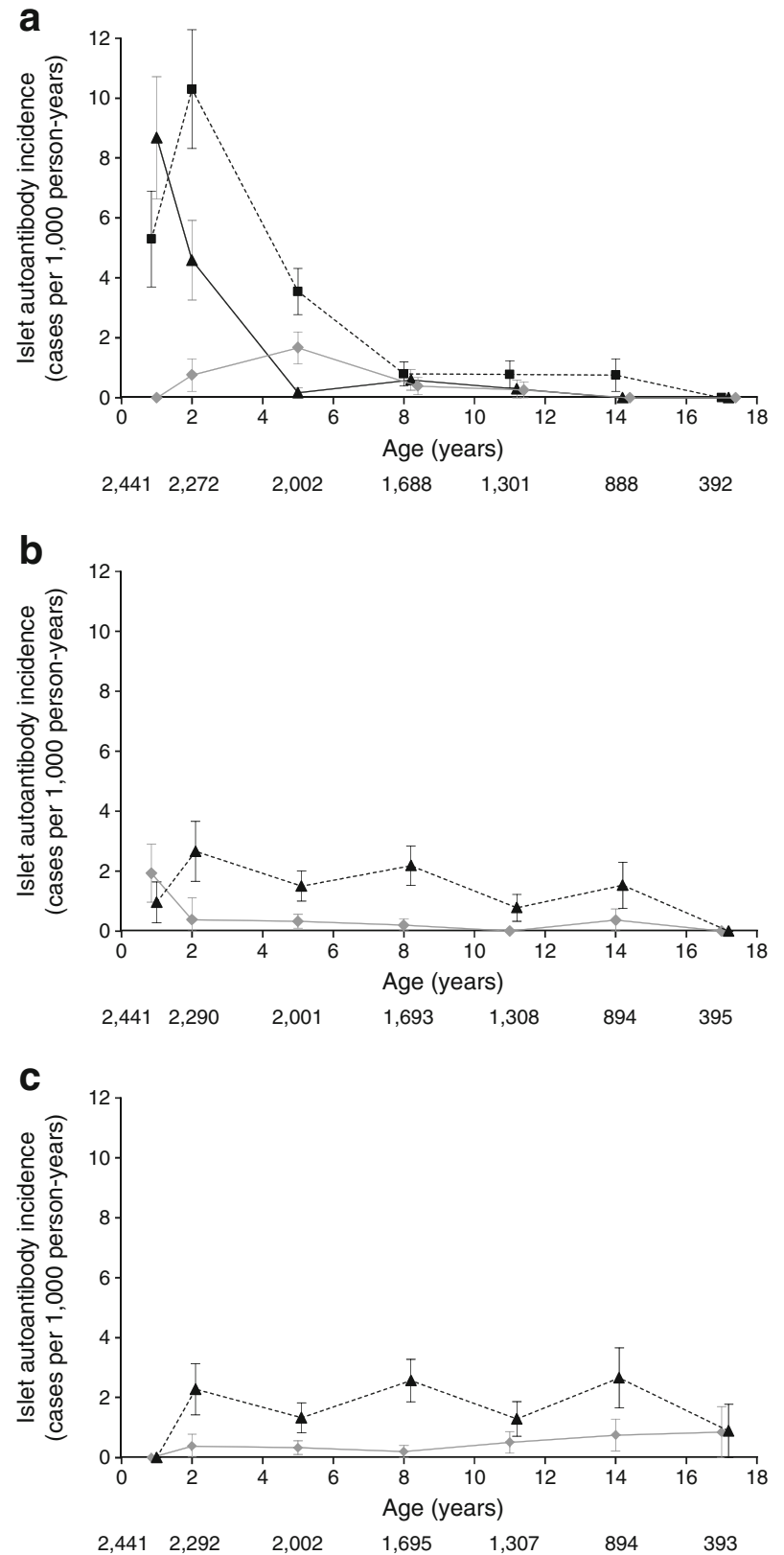

Fig. 2 Incidence (cases per 1,000 person-years) of specific islet autoantibody phenotypes, (a) Single IAA at seroconversion progressing to multiple islet autoantibodies (P1; black line; total positive = 35), single GADA at seroconversion progressing to multiple islet autoantibodies (P2; grey line; total positive $=15$ ) and multiple islet autoantibodies in the first positive sample (P3; black dashed line; total positive=35). (b) Single IAA with a high-affinity antibody phenotype (P4; grey line; total positive $=9)$ and single IAA with a low-affinity antibody phenotype (P5; black dashed line; total positive=36). (c) Single GADA positivity in RBA and ELISA (P6; grey line; total positive $=9$ ) and single GADA positivity in RBA only $(\mathrm{P} 7$; black dashed line; total positive $=38$ ). Error bars represent the SE; numbers under the $x$-axes indicate the total number of children still being followed at each time point

This was earlier than seen for those with the HLA DR3/DR4$D Q 8, D R 4-D Q 8 / D R 4-D Q 8$ and other HLA genotypes. 
Fig. 3 Incidence (cases per 1,000 person-years) of seroconversion to IAA preceding other islet autoantibodies or single highaffinity IAA (P1, P4; black line) and seroconversion to GADA preceding other islet autoantibodies or single GADA RBA and ELISA positivity (P2, P6; grey line) in children with the HLA DR3/DR4-DQ8 (a), DR4DQ8/DR4-DQ8 (b), HLA DR3/ $D R 3$ genotype (c) or other HLA genotypes (d). Error bars represent the SE; numbers under the $x$-axes indicate the total number of children with the HLA genotype still being followed at each time point a

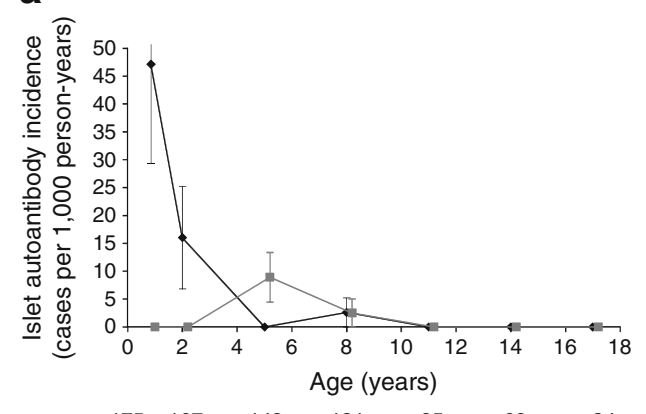

C

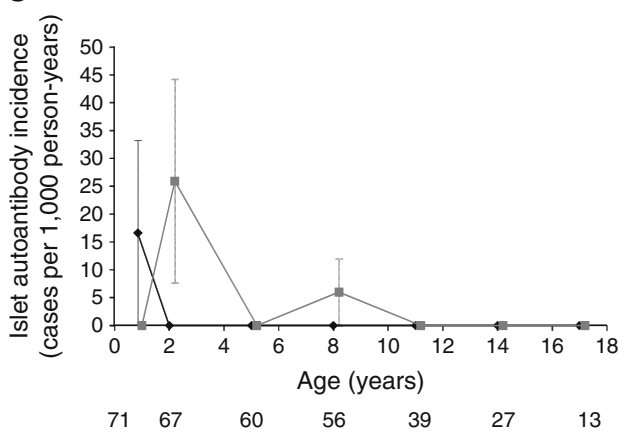

b

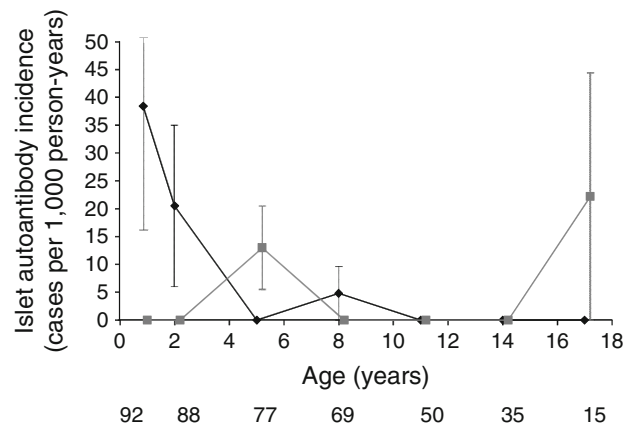

d

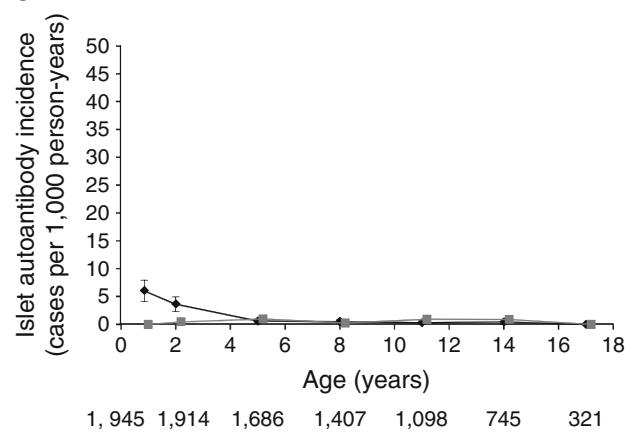

\section{Discussion}

The profile and antigenic specificity of islet autoantibodies at seroconversion varies between children. We categorised these profiles into phenotypes and showed temporal variation in the appearance of different islet autoantibody phenotypes. Autoimmunity that started with autoantibodies against insulin typically occurred sharply around 1 year of age and was associated with the $D R 4-D Q 8$ genotype, whereas autoimmunity directed against GAD occurred over a wide age range starting from age 2 years. These findings suggest that susceptibility to autoimmunity against specific beta cell autoantigens occurs at distinct age periods.

This study extends previous work from our own BABYDIAB study [3], the Finnish Type 1 Diabetes Prediction and Prevention (DIPP) study [14] and The Environmental Determinants of Diabetes in the Young (TEDDY) study [15]. It involved substantial follow-up of the cohort and uniquely characterised the islet autoantibodies at seroconversion and follow-up. A limitation was sampling intervals that were $>1$ year apart for many of the children. Therefore, seroconversion peak ages were rounded to the 9 month, 2 year and 5 year time points, and we have probably missed the initial single islet autoantibody stage in some children who were classified as having multiple islet autoantibodies in their first positive sample. We have not included single sample transient islet autoantibody-positive children; therefore, the total number of seroconversions is likely to be under-represented.
The predominant phenotypes observed were IAA preceding other autoantibodies (P1) and multiple islet autoantibodies in the first positive sample (P3), both of which were strongly associated with progression to clinical diabetes. The major islet autoantibody present in both phenotypes was IAA. Thus, initiation of autoimmunity against beta cell antigens including insulin often occurs within a window of susceptibility early in life. This was similar to findings of the Finnish DIPP study and the TEDDY study. Moreover, this period included the peak incidence of seroconversion to single high-affinity IAA. Of note, we observed that the appearance of IAA prior to other antibodies occurred earlier than IAA appearing together with other islet autoantibodies. This may reflect the time interval between sampling, but is consistent with an increased susceptibility period to autoimmunity against insulin at around 1 year of age. There was also a higher incidence of early insulin autoimmunity in children with $H L A$ DR4-DQ8/DR4-DQ8 and DR3/DR4-DQ8 genotypes, consistent with previous associations observed at diabetes onset [16-18]. These observations suggest that autoimmunity susceptibility linked to the HLA DR4-DQ8 genotype is poised for activation by environment.

In contrast, the susceptible period for autoimmunity against GAD as the first islet autoantibody was broader and occurred later compared with insulin, as observed in the TEDDY study [15]. Moreover, there was a link to the HLA DR3 allele as opposed to the $D R 4-D Q 8$ alleles. Indeed, children homozygous for the HLA DR3 genotype had a clear peak incidence of GAD first islet autoimmunity at around 2 years of age, while 
other children had a broader incidence pattern that started at around 2 years of age and was maximal at age 5 years. GADA were also heterogeneous in their binding profiles in different assays. We analysed the single GADA and found that more than half could not detect an N-terminally deleted antigen, suggesting that their epitopes were within the N-terminus, and that only a minority of single RBA-positive GADA were also positive in an ELISA shown to have high sensitivity at international workshops [19]. All single GADA-positive children who developed diabetes were GADA-positive in both the RBA and ELISA, suggesting that there may be prognostic value in measuring single GADA-positive samples with both assays.

In conclusion, our results show important differences in the temporal immunisation profiles for islet autoantigens. These may imply differences in the aetiology of islet autoimmunity in infancy. We suggest that there may be important benefits to categorising the autoantigen and the age period of autoimmunity development when investigating the genetic and environmental aetiology of type 1 diabetes. We also suggest that these findings could have implications for the timing of antigenspecific prevention using autoantigens such as insulin, proinsulin and GAD.

Acknowledgements We thank A. Knopff, S. Krause, A. Wosch, L. Schneider, S. Riethausen, J. Stock and K. Warncke of the Institute of Diabetes Research, Helmholtz Zentrum München, and Forschergruppe Diabetes, Klinikum rechts der Isar, Technische Universität München, Neuherberg, Germany, for data collection and expert technical assistance, and R. Puff, of the Institute of Diabetes Research, Helmholtz Zentrum München, and Forschergruppe Diabetes, Klinikum rechts der Isar, Technische Universität München, Neuherberg, Germany, for laboratory management. We thank V. Lampasona, San Raffaele Institute, Milan, Italy, for providing the $\mathrm{GADA}_{96-585}$ construct.

Funding The work was supported by grants from the Kompetenznetz Diabetes Mellitus, funded by the Federal Ministry of Education and Research (nos FKZ 01GI0805-07 and FKZ 01GI0805) and the JDRF (no. 17-2012-16), and funding from the German Federal Ministry of Education and Research to the German Center for Diabetes Research (DZD e.V.). EB is supported by the DFG Research Center and Cluster of Excellence - Center for Regenerative Therapies Dresden (FZ 111).

Duality of interest The authors declare that there is no duality of interest associated with this manuscript.

Contribution statement EZG and CW acquired and reviewed the data, undertook statistical analysis and interpretation of the results and drafted the manuscript. RC, CM and MS contributed to acquisition, analysis and interpretation and to writing the manuscript. $\mathrm{AB}$ contributed to the statistical analysis, interpreting the results and to writing the manuscript. PA contributed to data acquisition, analysis and interpretation and to writing the manuscript. EB made a major contribution to data analysis and interpretation, and contributed to writing the manuscript. A-GZ designed the study, is principal investigator of the BABYDIAB study, provided input to the analysis and contributed to writing the manuscript. All authors approved the final version of the manuscript. A-GZ takes responsibility for the integrity of the work as a whole.

\section{References}

1. Ziegler AG, Nepom GT (2010) Prediction and pathogenesis in type 1 diabetes. Immunity 32:468-478

2. Ziegler AG, Rewers M, Simell O et al (2013) Seroconversion to multiple islet autoantibodies and risk of progression to diabetes in children. JAMA 309:2473-2479

3. Ziegler AG, Bonifacio E, Babydiab-Babydiet Study Group (2012) Age-related islet autoantibody incidence in offspring of patients with type 1 diabetes. Diabetologia 55:1937-1993

4. Chmiel R, Giannopoulou EZ, Winkler C, Achenbach P, Ziegler AG, Bonifacio E (2015) Progression from single to multiple islet autoantibodies often occurs soon after seroconversion: implications for early screening. Diabetologia 58:411-413

5. Mayr A, Schlosser M, Grober N et al (2007) GAD autoantibody affinity and epitope specificity identify distinct immunization profiles in children at risk for type 1 diabetes. Diabetes 56:1527-1533

6. Achenbach P, Koczwara K, Knopff A, Naserke H, Ziegler AG, Bonifacio E (2004) Mature high-affinity immune responses to (pro)insulin anticipate the autoimmune cascade that leads to type 1 diabetes. J Clin Invest 114:589-597

7. Ziegler AG, Hummel M, Schenker M, Bonifacio E (1999) Autoantibody appearance and risk for development of childhood diabetes in offspring of parents with type 1 diabetes: the 2-year analysis of the German BABYDIAB Study. Diabetes 48:460-468

8. Hummel S, Pflüger M, Hummel M, Bonifacio E, Ziegler AG (2011) Primary dietary intervention study to reduce the risk of islet autoimmunity in children at increased risk for type 1 diabetes: the BABYDIET study. Diabetes Care 34:1301-1305

9. Lampasona V, Schlosser M, Mueller PW et al (2011) Diabetes antibody standardization program: first proficiency evaluation of assays for autoantibodies to zinc transporter 8. Clin Chem 57: 1693-1702

10. Schlosser M, Mueller PW, Törn C, Bonifacio E, Bingley PJ, Participating Laboratories (2010) Diabetes antibody standardization program: evaluation of assays for insulin autoantibodies. Diabetologia 53:2611-2620

11. Törn C, Mueller PW, Schlosser M, Bonifacio E, Bingley PJ, Participating Laboratories (2008) Diabetes antibody standardization program: evaluation of assays for autoantibodies to glutamic acid decarboxylase and islet antigen-2. Diabetologia 51:846-852

12. Puavilai G, Chanprasertyotin S, Sriphrapradaeng A (1999) Diagnostic criteria for diabetes mellitus and other categories of glucose intolerance: 1997 criteria by the expert committee on the diagnosis and classification of diabetes mellitus (ADA), 1998 Acta Diabetol 123 WHO consultation criteria, and 1985 WHO criteria. World Health Organization. Diabetes Res Clin Pract 44:21-26

13. Schenker M, Hummel M, Ferber K et al (1999) Early expression and high prevalence of islet autoantibodies for DR3/4 heterozygous and DR4/4 homozygous offspring of parents with type I diabetes: the German BABYDIAB study. Diabetologia 42:671-677

14. Parikka V, Näntö-Salonen K, Saarinen M et al (2012) Early seroconversion and rapidly increasing autoantibody concentrations predict prepubertal manifestation of type 1 diabetes in children at genetic risk. Diabetologia 55:1926-1936

15. Krischer JP, Lynch KF, Schatz DA et al (2015) The six-year incidence of diabetes associated autoantibodies in genetically at-risk children: the TEDDY study. Diabetologia 58:980-987

16. Walter M, Albert E, Conrad M et al (2003) IDDM2/insulin VNTR modifies risk conferred by IDDM1/HLA for development of Type 1 diabetes and associated autoimmunity. Diabetologia 46:712-720

17. Valdes AM, Erlich HA, Carlson J, Varney M, Moonsamy PV, Noble JA (2012) Use of class I and class II HLA loci for predicting 
age at onset of type 1 diabetes in multiple populations. Diabetologia 55:2394-2401

18. Awa WL, Boehm BO, Kapellen T et al (2010) HLA-DR genotypes influence age at disease onset in children and juveniles with type 1 diabetes mellitus. Eur J Endocrinol 163:97-104
19. Williams AJK, Lampasona V, Schlosser M et al (2015) Detection of antibodies directed to the $\mathrm{N}$-terminal region of GAD is dependent on assay format and contributes to differences in the specificity of GAD autoantibody assays for type 1 diabetes. Diabetes. doi:10. 2337/db14-1693 